\title{
Research of oxygen concentration information detecting method with LIA
}

\author{
Dianhong $\mathrm{Yu}^{\mathrm{a}}$, Zhen Guo and Lin Li \\ School of Mechanical \& Instrumental Engineering Xi'an University of Technology, Xi'an, Shaanxi/710048, China
}

\begin{abstract}
A method of detecting weak signals by two lock-in amplifiers is introduced to detect oxygen concentration as a weak signal, in the process of detecting oxygen concentration based on TDLAS. In this paper, the working principle of lock-in amplifier is described, and the principle and characteristics of detecting weak signals with two lock-in amplifiers are analyzed. The second harmonic signal is selected as the main extraction target. By simulating and analyzing the extraction process of signal with different phase, the feasibility and importance in improving detection accuracy are verified.
\end{abstract}

Keywords: weak signals; lock-in amplifier; oxygen concentration detection; TDLAS.

\section{Introduction}

The development and improvement of industrialization level in China has brought not only great progress in people's living standards and socio-economic situation but also more and more prominent air pollution problems. Air pollution in China belongs to soot pollution; the main pollutants are smoke, $\mathrm{SO}_{2}$, nitrogen oxides and $\mathrm{CO}$, and so on. As a combustion-supporting gas, the concentration of $\mathrm{O}_{2}$ directly affects the combustion efficiency and sufficiency and also determines the type and content of the gas after combustion [1]. If the concentration of $\mathrm{O}_{2}$ is insufficient, incomplete combustion will be caused, which will produce $\mathrm{CO}$ and other harmful gases that not only pollute the environment, but also damage human health. Therefore, to monitor the oxygen concentration in real-time not only helps to improve combustion efficiency and reduce the emission of pollutants, but also is directly related to personal safety and security of the production environment.

At present, with the development of TDLAS and the breakthrough in DFB, VCSEL and other semiconductor laser, a new path for the detection of oxygen concentration has opened up. TDLAS, which combines with wavelength modulation technique [2], is widely used in air pollution monitoring, biomedical, aerospace and other fields. It is the weak signal detection for oxygen concentration and is mainly based on autocorrelation and cross-correlation which can extract the useful signal submerged in the big noise effectively and helps to improve the SNR. Lock-in amplifier [3], which is the electronic equipment designed by the principle, is widely used in all fields of scientific research. In this paper, based on TDLAS combined with lock-in amplifier, the signal containing the concentration of oxygen will be analyzed and the signal distilling method will be studied.

\footnotetext{
a Corresponding author : yudh@xaut.edu.cn
} 


\section{Theory}

\subsection{Principle of oxygen concentration detection based on TDLAS}

The absorption spectrum will be formed after the laser energy is absorbed by frequency selection. This principle is used for TDLAS to measure the gas concentration. It is a laser monitoring technique based on gas absorption spectroscopy. That is, if the frequency of the laser passing through the measured gas is equal to the center frequency of the measured gas, the photon with the frequency will be absorbed that leads to laser intensity attenuation. It obeys Beer-Lambert's law [4]:

$$
I(t)=I_{0}(t) \exp \left[-S(T) g\left(v, v_{0}\right) P C L\right]
$$

where, $\mathrm{I}(\mathrm{t})$ and $\mathrm{I} 0(\mathrm{t})$ are the output and input light intensity, $\mathrm{S}(\mathrm{t})$ is intensity of the absorption line, $g(v, v 0)$ is the line shape, $P$ is the gas pressure, $C$ is the gas concentration and $L$ represents interaction length. Under atmosphere pressure and temperature, the line shape is given by Lorentzian distribution:

$$
g\left(v, v_{0}\right)=\frac{1}{2 \pi} \frac{\Delta v_{N}}{\left(v-v_{0}\right)^{2}+\left(\frac{\Delta v_{N}}{2}\right)^{2}}
$$

where, $v 0$ and $\Delta v \mathrm{~N}$ represent the central frequency and the full width of the absorption line.

When the laser driving current is modulated, the frequency and the intensity of the output laser light can be written as

$$
\begin{gathered}
v=v_{0}+v_{m} \sin (2 \pi f t) \\
I_{0}(v)=I_{0}[1+\eta \sin (2 \pi f t)]
\end{gathered}
$$

where, $v$ m represents the amplitude of the frequency modulation and $\eta$ is intensity modulation index and $\mathrm{f}$ is the frequency of modulation.

When, gas absorption and the intensity modulation are very small, I(t) may be approximated as

$$
m=2 v_{m} / \Delta v_{N}
$$

where, $m$ is represented by .

Then,with expanding the Eq.(5) using Taylor series, the 2nd Fourier components of absorption signalat center of the absorption line is proportional to the concentration. This is expressed as

$$
I_{2 f} \propto I_{0} S(T) g\left(v, v_{0}\right) C P L
$$

Therefore, the concentration of oxygen can be obtained by extracting the second harmonic signal.

\subsection{Working principle of lock-in amplifier}

Lock-in amplifier is a kind of analog Fourier transforms [5] which is designed by using the principle of cross-correlation. The schematic is shown in Figure1. The signal to be measured is input to lock-in amplifier and mixed with a reference signal after amplified and filtered by bandpass filter. Then, the result is filtered by low pass filter and outputted. 


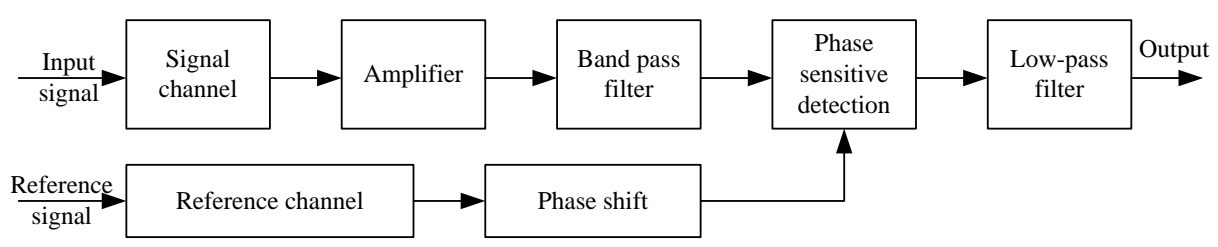

Figure1. The principle of lock-in amplifier

It is assumed that the signal to be measured is represented by $s(t)=A \sin (\omega t+\phi)$, and then the input signal and reference signal can be written as

$$
\begin{gathered}
x(t)=s(t)+n(t)=A \sin (\omega t+\phi)+n(t) \\
y(t)=B \sin [w(t+\tau)]
\end{gathered}
$$

where, $\mathrm{n}(\mathrm{t})$ represents noise and $\tau$ is the time shifts. Then, the cross-correlation function is expressed as

$$
R_{x y}=\lim _{T \rightarrow \infty} \frac{1}{T} \int_{0}^{T} x(t) y(t) d t=\frac{A B}{2} \cos (\omega t+\phi)+R_{x n}(\tau)
$$

where, $\operatorname{Rxn}(\mathrm{t})=0$ which is the cross-correlation function of $\mathrm{x}(\mathrm{t})$ and $\mathrm{n}(\mathrm{t})$ because they are uncorrelated. Thus, $\operatorname{Rxn}(\tau)$ is proportional to the amplitude of $s(t)$. So, it's feasible to filter noise by cross correlating operation.

Phase-sensitive detector is the core part of lock-in amplifier, by which the input signal $\mathrm{us}(\mathrm{t})=\mathrm{U} \sin (2 \pi \mathrm{f} 1 \mathrm{t}+\phi 1)$ is mixed with the reference signal $\mathrm{xr}(\mathrm{t})=\mathrm{R} \sin (2 \pi \mathrm{f} 2 \mathrm{t}+\phi 2)$. After the correlating calculation, the output signal can be derived as

$$
u(t)=\frac{U R}{2} \cos \left[2 \pi\left(f_{1}-f_{2}\right) t+\left(\phi_{1}-\phi_{2}\right)\right]-\frac{U R}{2} \cos \left[2 \pi\left(f_{1}+f_{2}\right) t+\left(\phi_{1}+\phi_{2}\right)\right]
$$

where, $\mathrm{f} 1$ and $\mathrm{f} 2$ are the frequencies of the input signal and the reference signal. When, $\mathrm{f} 1$ is equal to $\mathrm{f} 2$, and the frequency-multiplier signal is filtered by lowpass filter, $\mathrm{u}(\mathrm{t})$ can be written as

$$
u(t)=\frac{U R}{2} \cos \left(\phi_{1}-\phi_{2}\right)
$$

Therefore, the signal demodulated by lock-in amplifier will be proportional to the input signal if the phase of the input signal is the same as the reference signal.

\subsection{Method of extracting harmonic signal}

From above, we know that the phase of input signal must be the same as the phase of reference signal when using the lock-in amplifier to demodulate the weak signal received by detector. But in fact, it is difficult to assure that. In view of this, we use two lock-in amplifiers with phase difference of $90^{\circ}$ of reference signals to eliminate the effect of the phase difference[6]. In this way, the two output signals can be written as

$$
u_{x}(t) \propto U \cos \theta
$$




$$
u_{y}(t) \propto U \cos \left(\theta+90^{\circ}\right)=U \sin \theta
$$

where, $\theta=\phi 1-\phi 2$ is the phase difference between the signal to be measured and the reference signal. Then, the synthetic amplitude of $\mathrm{ux}(\mathrm{t})$ and uy(t) can be expressed as

$$
R \propto \sqrt{u_{x}(t)^{2}+u_{y}(t)^{2}}=\sqrt{(U \cos \vartheta)^{2}+(U \sin \theta)^{2}}=U
$$

Therefore, the synthetic amplitude is proportional to the amplitude of the input signal and not affected by the phase difference. So, this method can improve the sensitivity.

The schematic diagram of harmonic signal extraction process is shown in Figure2. Current from detector is converted to voltage, and then, filtered by bandpass filter after being amplified. Then, the signal with phase shifted is sent to two lock-in amplifiers. In order to extract the second harmonic signal to be measured, the frequency of the reference signal to be sent into the lock-in amplifier is 2 times as much as the frequency of the modulated signal. Where, the differential phase between the two reference signals is $90^{\circ}$. By synthetising $\mathrm{ux}(\mathrm{t})$ and $\mathrm{uy}(\mathrm{t})$, the second harmonic signal can be extracted.

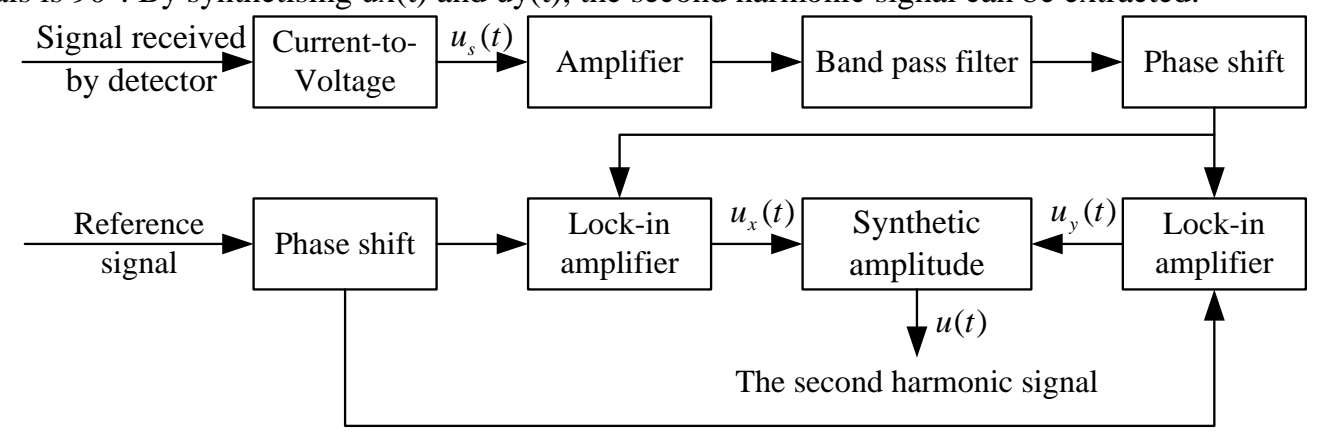

Figure2. The schematic diagram of harmonic signal extraction process

\section{Simulation}

Based on the technology of TDLAS, the processes of gas absorption and harmonic signal extraction are simulated by Matlab. As shown in Figure3 (a), laser scanning signal using a $1 \mathrm{~Hz}$ sawtooth-wave signal and laser modulating signal using a $200 \mathrm{~Hz}$ sine-wave signal are superimposed through an added. And then, the synthesized signal scans and modulates the laser. After that, the laser is absorbed by oxygen whose line shape is given by Lorenzian distribution. It's assumed that the amplitude is 0.4 , the central frequency is 0.5 and the half width of the absorption is 0.05 . The result is shown in Figure3 (b). Subsequently, based on Lambert-Beer law, the signal received by detector can be described as shown in Figure3(c). It can be seen that an obvious depression is appeared at the central frequency in the signal received by detector, which shows that the light intensity is absorbed and weakened obviously. 

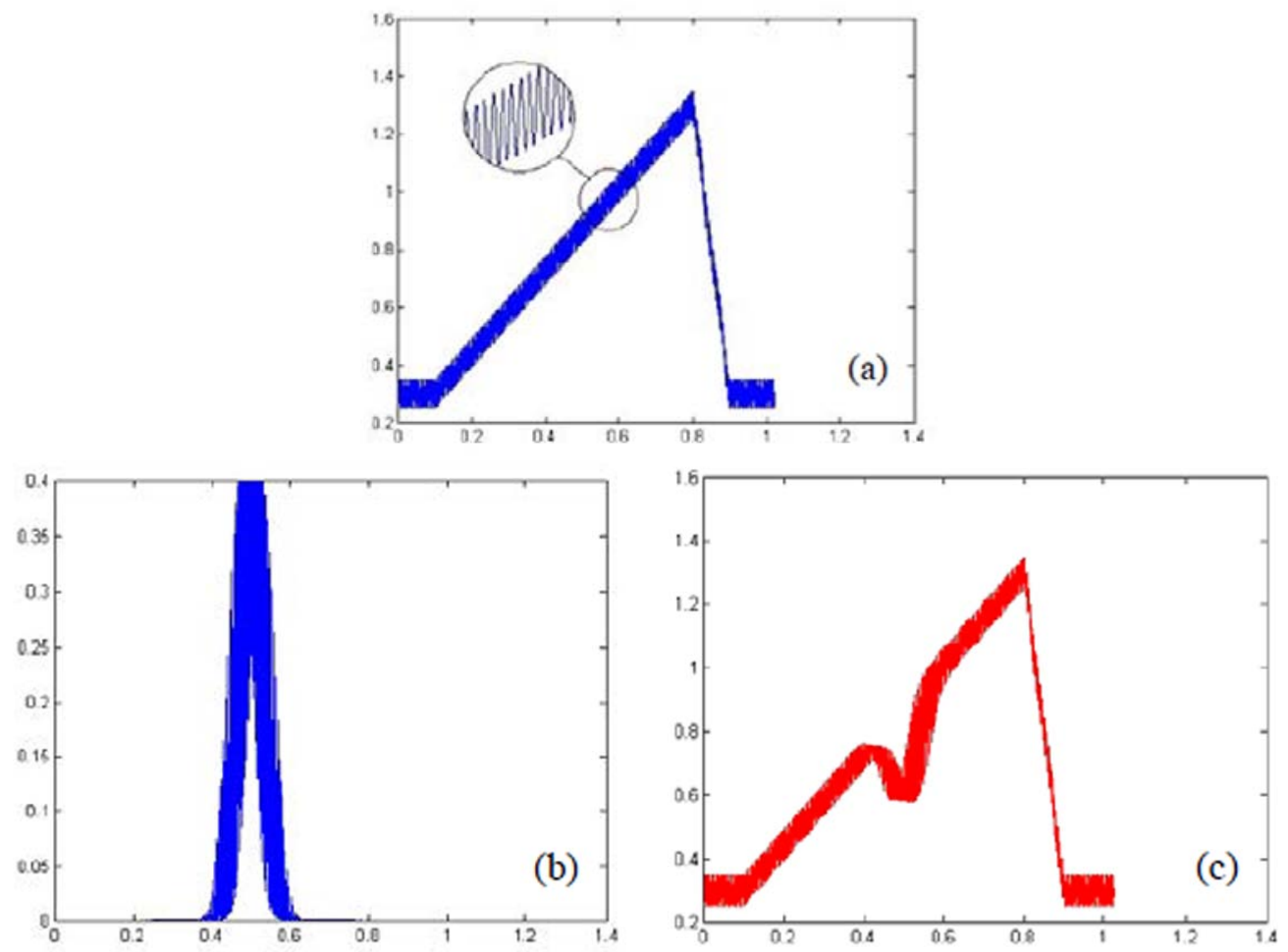

Figure3. The processes of gas absorption, (a) driving signal of laser source, (b) linetype of gas absorption, and (c) signal received by detector.

According to the method of extracting the second harmonic signal, a $400 \mathrm{~Hz}$ sinusoidal signal and a $400 \mathrm{~Hz}$ cosine signal are used as reference signals. While simulating, the phase difference between signal received by detector and reference signal is respectively $0^{\circ}, 30^{\circ}, 45^{\circ}$ and $90^{\circ}$. And the second harmonic signals are shown in Figure4. Where, the "Signal 1" and "Signal 2" are the second harmonic signals extracted by two lock-in amplifiers, and the "synthesis" is the synthesized signal. As can be seen from the comparison among Figure4(a)- (d), with the changes of the phase difference, amplitudes of the two harmonic signals are changed, expect for the synthesized signal. So detection accuracy is assured.
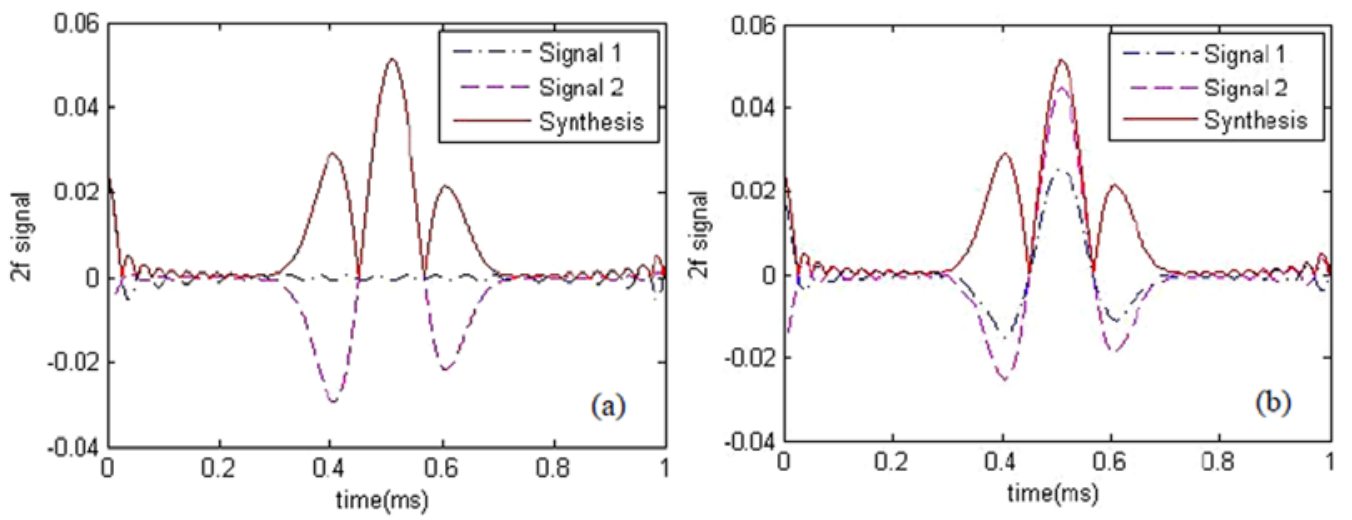

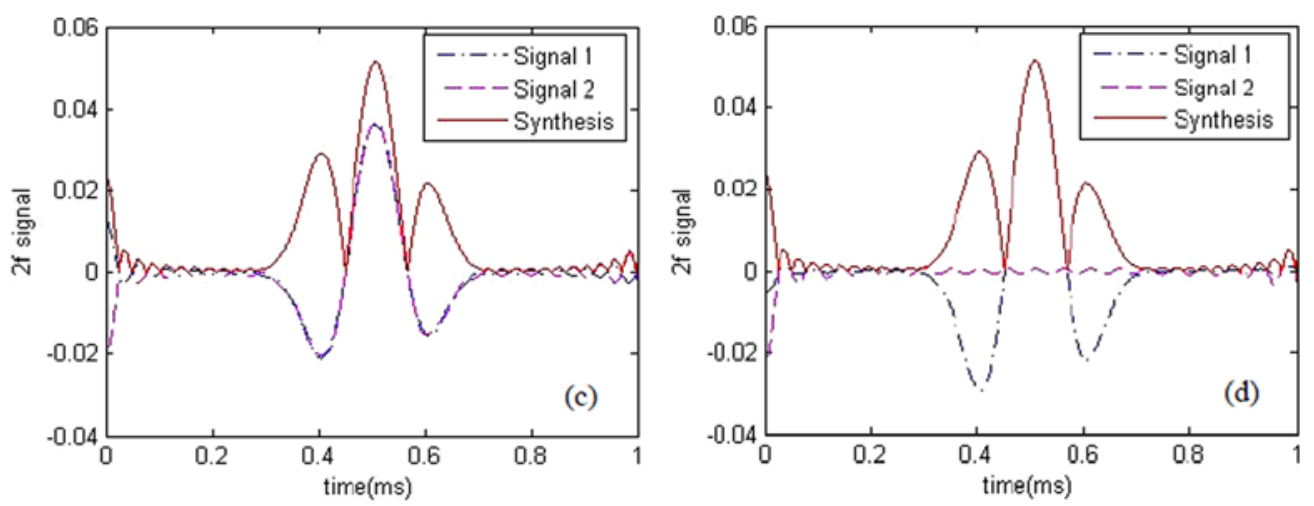

Figure 4. Second harmonic signal in different phase difference, (a) $0^{\circ}$ phasedifference, (b) $30^{\circ}$ phase difference, (c) $45^{\circ}$ phase difference, and (d) $90^{\circ}$ phase difference.

\section{Conclusion}

Based on TDLAS technology, wavelength modulation combined with lock-in amplifier technique provided an effective method for gas concentration detection. The detection of weak signal was realized by using lock-in amplifier based on correlation theory, which could extract the target signals from noise effectively. So, the SNR and detection sensitivity were improved. The problem of the phase difference caused by inaccurate regulation and circuit effects had been solved by using two lock-in amplifiers to extract the harmonic signal, which could improve the accuracy of detection and obtain the more accurate concentration information.

\section{Acknowledgment}

The authors gratefully acknowledges the support of the National Natural Science Foundation of China (Project Grant No. 51175421) and the Shaanxi Province Industrial Science and technology project (Project Grant No. 2015GY100)

\section{References}

1. YAN Jie, ZHAI Chang, WANG Xiao-niu, et al. The industrial process Oxygen analyzer based on tdlas[J]. China Instrumentation, 2014(9): 67-71.

2. ZHANG Zhi-rong, DING Zong-fu, DING Zong-ling, et al. Application of tunable diode laser absorption spectroscopy on monitoring of Oxygen concentration in industry control [J]. Ludong University Journal(Natural Science Edition), 2008, 24(2): 142-144.

3. JIAO Bin-liang, LI Su-jing. Lock-in amplifier and its correlation detection thorough analysis[J]. Electronic Technology, 2008, 45(1): 110-112.

4. CHEN Jiu-ying, JIANGUO L, HE Ya-bai, et al. Scanning frequency optimization of laser absorption spectroscopy [J]. ACTA Optica Sinica, 2013, 33(2): 2300023-2300031.

5. NI Jia-sheng, LIU Tong-yu, WANG Chang, et al. Design of a Lock-in amplifier circuit and its application in a trace gas sensor [J]. Shandong Science, 2009, 22(5): 48-52,56.

6. SU Xin, LUO W, MA Chao, et al. A design method for the dual phase Lock-in amplifier[J]. Electronic Science and Technology, 2012, 25(3): 75-77,81. 